\title{
INTRINSICALLY LIPSCHITZ FUNCTIONS WITH NORMAL TARGET IN CARNOT GROUPS
}

\author{
Gioacchino Antonelli and Andrea Merlo
}

\author{
Scuola Normale Superiore \\ Piazza dei Cavalieri, 7, 56126 Pisa, Italy; gioacchino.antonelli@sns.it \\ Universitá di Pisa \\ Largo Bruno Pontecorvo, 5, 56126 Pisa, Italy; andrea.merlo@sns.it
}

\begin{abstract}
We provide a Rademacher theorem for intrinsically Lipschitz functions $\phi: U \subseteq$ $\mathbf{W} \rightarrow \mathbf{L}$, where $U$ is a Borel set, $\mathbf{W}$ and $\mathbf{L}$ are complementary subgroups of a Carnot group, where we require that $\mathbf{L}$ is a normal subgroup. Our hypotheses are satisfied for example when $\mathbf{W}$ is a horizontal subgroup. Moreover, we provide an area formula for this class of intrinsically Lipschitz functions.
\end{abstract}

\section{Introduction}

In the last years there has been an increasing interest towards Geometric Measure Theory in the non-smooth setting, and in particular in the setting of sub-Riemannian Carnot groups for what concers the notion of rectifiability. This line of research was initiated by the result in [1] in which the authors proved that the first Heisenberg group $\mathbf{H}^{1}$ is not $k$-rectifiable, according to Federer's definition (see [7]), for $k \geq 2$.

In this note we focus on the notions of intrinsically Lipschitz function and intrinsically differentiable function in the setting of Carnot groups. We refer the reader to [9] for a wide study of the notion of intrinsically Lipschitz function and to [5] for some recent developments. The notion of intrinsically differentiable function has been introduced in [11] and then widely studied in the last years: we refer the reader to $[2,4,12,8,3]$ for some developments.

One of the main open questions in this area of research is whether a Rademacher type theorem holds. Namely, is it true that every intrinsically Lipschitz function between complementary subgroups of a Carnot group is intrinsically differentiable almost everywhere? Some first answers have been given in $[8,12]$ in the setting of Carnot groups $\mathbf{G}$ of type $\star$, i.e., a class strictly larger than Carnot groups of step 2, and for maps $\phi: U \subseteq \mathbf{W} \rightarrow \mathbf{L}$, where $U$ is open and $\mathbf{W}$ and $\mathbf{L}$ are complementary subgroups of $\mathbf{G}$, with $\mathbf{L}$ horizontal and one-dimensional. Very recently, by making use of currents, the author of [19] has proved the Rademacher theorem for intrinsically Lipschitz maps between complementary subgroups of any dimension in the Heisenberg groups $\mathbf{H}^{n}$.

In this note we prove that a Rademacher type theorem with normal target is true in arbitrary Carnot groups. We provide also an area formula for maps that satisfy our hypotheses.

Theorem 1.1. Let $\mathbf{W}$ and $\mathbf{L}$ be two complementary subgroups of a Carnot group $\mathbf{G}$. Let us assume that $\mathbf{L}$ is normal and let $U \subseteq \mathbf{W}$ be a Borel set. Let

https://doi.org/10.5186/aasfm.2021.4638

2020 Mathematics Subject Classification: Primary 53C17, 22E25, 28A75, 49Q15, 26 A16.

Key words: Carnot groups, intrinsically Lipschitz functions, Rademacher theorem, area formula. 
$\phi: U \subseteq \mathbf{W} \rightarrow \mathbf{L}$ be an intrinsically Lipschitz function. Let $\Phi: U \rightarrow \mathbf{G}$ be the graph map of $\phi$, i.e., $\Phi(w):=w \cdot \phi(w)$ for every $w \in U$.

Then $\mathbf{W}$ is a Carnot subgroup of $\mathbf{G}$; $\phi$ is intrinsically differentiable $\mathcal{H}^{k}\llcorner\mathbf{W}$ almost everywhere on $U$, where $k:=\operatorname{dim}_{H}(\mathbf{W})$; the map $\Phi$ is Pansu differentiable $\mathcal{H}^{k}\llcorner\mathbf{W}$-almost everywhere on $U$, and the following area formula holds:

$$
\mathcal{H}^{k}(\Phi(V))=\int_{V} J\left(d \Phi_{x}\right) d \mathcal{H}^{k}(x), \quad \text { for every Borel set } V \subseteq U,
$$

where $d \Phi_{x}$ is the Pansu differential of $\Phi$ at $x \in V$ and $J\left(d \Phi_{x}\right)$ is its Jacobian, see (14).

We stress that the hypotheses of Theorem 1.1 are satisfied for example when $\mathbf{W}$ is horizontal. Thus our theorem holds for intriniscally Lipschitz horizontal surfaces in arbitrary Carnot groups. We stress also that an area formula for maps that parametrizes $C_{H}^{1}$-submanifolds, that is a more restrictive condition than being intrinsically Lipschitz, has been recently obtained in [13, Theorem 1.1]. In the latter reference the authors call $C_{\mathrm{H}^{-}}^{1}$ submanifold a subset of a Carnot group $\mathbf{G}$ that is, locally around every point, the zero-level set of a $C_{\mathrm{H}}^{1}$ function $f$ defined on an open subset of $\mathbf{G}$ with value in a Carnot group $\mathbf{G}^{\prime}$, and such that moreover the Pansu differential $D f$ is surjective and $\operatorname{Ker}(D f)$ splits $\mathbf{G}$.

The proof of Theorem 1.1 is considerably simpler than the proof of a lowcodimensional Rademacher theorem because in that case it may not be true, as in our case, that the intrinsic Lipschitz property of $\phi$ reads as the graph map $\Phi$ being Lipschitz (see Remark 3.3). In fact we apply Pansu-Rademacher theorem and Magnani's area formula to deduce that the graph map $\Phi$ is Pansu differentiable almost everywhere and the area formula holds. Thus in order to complete the proof of Theorem 1.1, we are left to relate the Pansu differentiability of the graph map $\Phi$ with the intrinsic differentiability of the map $\phi$, and this is done in Proposition 3.2.

Acknowledgments. The first author is partially supported by the European Research Council (ERC Starting Grant 713998 GeoMeG 'Geometry of Metric Groups'). The authors are grateful to Enrico Le Donne for several suggestions that led to an improvement of this note. They are also grateful to Sebastiano Don for precious comments.

\section{Preliminaries}

For the basic terminology on Carnot groups and graded groups we refer the reader to [14]. Every Carnot group in this note will come together with a stratification $\operatorname{Lie}(\mathbf{G})=V_{1} \oplus \cdots \oplus V_{s}$. We recall some notation. Every graded group has a oneparameter family of dilations that we denote by $\left\{\delta_{\lambda}: \lambda>0\right\}$. We will indicate with $\delta_{\lambda}$ both the dilation of factor $\lambda$ on the group and its differential.

Given a graded group $\mathbf{G}$, we fix a homogeneous norm $\|\cdot\|$ on $\mathbf{G}$, which is unique up to bi-Lipschitz equivalence. Moreover, by [10, Theorem 5.1] on every Carnot group there exists a slight variation of the anisotropic homogeneous norm that induces a left-invariant homogeneous distance $d$. We eventually fix this particular homogeneous norm from now on. We also recall that with homogeneous homomorphism we mean a homomorphism that commutes with $\delta_{\lambda}$, for every $\lambda>0$. We now deal with complementary subgroups and maps between them.

Definition 2.1. (Complementary subgroups) Given a Carnot group G with identity $e$, we say that two subgroups $\mathbf{W}$ and $\mathbf{L}$ are complementary subgroups in 
$\mathbf{G}$ if they are homogeneous, i.e., closed under the action of $\delta_{\lambda}$ for every $\lambda>0$, $\mathbf{G}=\mathbf{W} \cdot \mathbf{L}$, and $\mathbf{W} \cap \mathbf{L}=\{e\}$.

Given two complementary subgroups $\mathbf{W}$ and $\mathbf{L}$ in a Carnot group $\mathbf{G}$, we denote the projection maps from $\mathbf{G}$ onto $\mathbf{W}$ and onto $\mathbf{L}$ by $\pi_{\mathbf{W}}$ and $\pi_{\mathbf{L}}$, respectively. Defining $g_{\mathbf{W}}:=\pi_{\mathbf{W}} g$ and $g_{\mathbf{L}}:=\pi_{\mathbf{L}} g$ for any $g \in \mathbf{G}$, one has

$$
g=\left(\pi_{\mathbf{W}} g\right) \cdot\left(\pi_{\mathbf{L}} g\right)=g_{\mathbf{W}} \cdot g_{\mathbf{L}}
$$

We recall the following basic terminology: a horizontal subgroup of a Carnot group $\mathbf{G}$ is a homogeneous subgroup of it that is contained in $\exp \left(V_{1}\right)$, where $V_{1}$ is the first layer of the stratification of $\operatorname{Lie}(\mathbf{G})$; a Carnot subgroup $\mathbf{W}$ of a Carnot group $\mathbf{G}$ is a homogeneous subgroup of it such that the first layer $V_{1}(\mathbf{W}):=V_{1} \cap \operatorname{Lie}(\mathbf{W})$ of the grading of $\operatorname{Lie}(\mathbf{W})$ inherited from the stratification of $\operatorname{Lie}(\mathbf{G})$ is the first layer of a stratification of Lie( $\mathbf{W})$. Before going on we add the following remark, which will be important in the proof of Theorem 1.1.

Remark 2.1. (The complement of a normal subgroup is Carnot) We claim that if $\mathbf{W}$ and $\mathbf{L}$ are two homogeneous complementary subgroups of a Carnot group $\mathbf{G}$, and $\mathbf{L}$ is normal, then $\mathbf{W}$ is a Carnot subgroup of $\mathbf{G}$. First we claim that Lie( $\mathbf{L})$ is an ideal of $\operatorname{Lie}(\mathbf{G})$. Indeed, if we set Ad to be the adjoint operator on $\mathbf{G}$, see [6, page 12], for every $X \in \operatorname{Lie}(\mathbf{G})$ and $Y \in \operatorname{Lie}(\mathbf{L})$ we have $[X, Y]=\left.\frac{d}{d t}\right|_{t=0} \operatorname{Ad}_{\exp (t X)} Y \in \operatorname{Lie}(\mathbf{L})$, where the last inclusion follows from the fact that $\operatorname{Ad}_{\exp (t X)} Y \in \operatorname{Lie}(\mathbf{L})$ for every $t>0$, because $\mathbf{L}$ is normal. Therefore, if $\operatorname{Lie}(\mathbf{G})=V_{1} \oplus \cdots \oplus V_{s}$ is the stratification of $\operatorname{Lie}(\mathbf{G})$, in order to prove that $\mathbf{W}$ is a Carnot subgroup of $\mathbf{G}$ it is enough to prove that

$$
V_{i+1} \cap \operatorname{Lie}(\mathbf{W}) \subseteq\left[V_{1} \cap \operatorname{Lie}(\mathbf{W}), V_{i} \cap \operatorname{Lie}(\mathbf{W})\right],
$$

for every $i=1, \ldots, s$; indeed the other inclusion $V_{i+1} \cap \operatorname{Lie}(\mathbf{W}) \supseteq\left[V_{1} \cap \operatorname{Lie}(\mathbf{W}), V_{i} \cap\right.$ $\operatorname{Lie}(\mathbf{W})$ ] follows from the fact that $\operatorname{Lie}(\mathbf{W})$ is a Lie subalgebra of $\operatorname{Lie}(\mathbf{G})$ because $\mathbf{W}$ is a homogeneous subgroup of $\mathbf{G}$. The inclusion (2) readily follows from the following observation, extended linearly: if $p_{1} \in V_{1}$ and $p_{i} \in V_{i}$ are such that $\left[p_{1}, p_{i}\right] \in \operatorname{Lie}(\mathbf{W})$, then $\left[\pi_{\mathrm{Lie}(\mathbf{W})} p_{1}, \pi_{\mathrm{Lie}(\mathbf{W})} p_{i}\right]=\left[p_{1}, p_{i}\right]$, where $\pi_{\mathrm{Lie}(\mathbf{W})}$ is the projection associated to the splitting $\operatorname{Lie}(\mathbf{G})=\operatorname{Lie}(\mathbf{W}) \oplus \operatorname{Lie}(\mathbf{L})$. Indeed, $\left[p_{1}, p_{i}\right]+\operatorname{Lie}(\mathbf{L})=\left[\pi_{\operatorname{Lie}(\mathbf{W})} p_{1}+\right.$ $\left.\pi_{\operatorname{Lie}(\mathbf{L})} p_{1}, \pi_{\operatorname{Lie}(\mathbf{W})} p_{i}+\pi_{\operatorname{Lie}(\mathbf{L})} p_{i}\right]+\operatorname{Lie}(\mathbf{L})=\left[\pi_{\operatorname{Lie}(\mathbf{W})} p_{1}, \pi_{\operatorname{Lie}(\mathbf{W})} p_{i}\right]+\operatorname{Lie}(\mathbf{L})$, since Lie $(\mathbf{L})$ is an ideal. From the latter equality the last claim follows since $\left[p_{1}, p_{i}\right] \in \operatorname{Lie}(\mathbf{W})$.

We now describe what is the parametrizing function of some translation of the intrinsic graph of a function. Unless anything different is declared, we eventually fix from now on two arbitrary complementary subgroups $\mathbf{W}$ and $\mathbf{L}$ of a Carnot group G.

Definition 2.2. (Intrinsic graph) Given a function $\phi: U \subseteq \mathbf{W} \rightarrow \mathbf{L}$, we define the intrinsic graph of $\phi$ as follows:

$$
\operatorname{graph}(\phi):=\{\Phi(w):=w \cdot \phi(w): w \in U\}=: \Phi(U) .
$$

Definition 2.3. (Intrinsic translation of a function) Given a function $\phi: U \subseteq$ $\mathbf{W} \rightarrow \mathbf{L}$, we define, for every $q \in \mathbf{G}$,

$$
U_{q}:=\left\{a \in \mathbf{W}: \pi_{\mathbf{W}}\left(q^{-1} \cdot a\right) \in U\right\},
$$

and $\phi_{q}: U_{q} \subseteq \mathbf{W} \rightarrow \mathbf{L}$ by setting

$$
\phi_{q}(a):=\left(\pi_{\mathbf{L}}\left(q^{-1} \cdot a\right)\right)^{-1} \cdot \phi\left(\pi_{\mathbf{W}}\left(q^{-1} \cdot a\right)\right) .
$$


For the following proposition we also refer the reader to [9, Proposition 2.21 and Remark 2.23].

Proposition 2.1. Given a function $\phi: U \subseteq \mathbf{W} \rightarrow \mathbf{L}$, and $q \in \mathbf{G}$, the following facts hold:

(a) $\operatorname{graph}\left(\phi_{q}\right)=q \cdot \operatorname{graph}(\phi)$;

(b) if $\mathbf{L}$ is normal one gets that, for all $a \in U$,

$$
\pi_{\mathbf{W}}\left(q^{-1} \cdot a\right)=q_{\mathbf{W}}^{-1} \cdot a, \quad \pi_{\mathbf{L}}\left(q^{-1} \cdot a\right)=a^{-1} \cdot q_{\mathbf{W}} \cdot q_{\mathbf{L}}^{-1} \cdot q_{\mathbf{W}}^{-1} \cdot a,
$$

and thus

$$
\phi_{q}(a)=a^{-1} \cdot q_{\mathbf{W}} \cdot q_{\mathbf{L}} \cdot q_{\mathbf{W}}^{-1} \cdot a \cdot \phi\left(q_{\mathbf{W}}^{-1} \cdot a\right), \quad U_{q}=q_{\mathbf{W}} \cdot U ;
$$

Proof. The proof of (a) directly follows from (3), which yields

$$
a \cdot \phi_{q}(a)=q \cdot \pi_{\mathbf{W}}\left(q^{-1} \cdot a\right) \cdot \phi\left(\pi_{\mathbf{W}}\left(q^{-1} \cdot a\right)\right), \quad \forall a \in U_{q} .
$$

Let us prove (b). Since $\mathbf{L}$ is normal, the following holds:

$$
a^{-1} \cdot q_{\mathbf{W}} \cdot q_{\mathbf{L}}^{-1} \cdot q_{\mathbf{W}}^{-1} \cdot a=a^{-1} \cdot q_{\mathbf{W}} \cdot q_{\mathbf{L}}^{-1} \cdot\left(a^{-1} \cdot q_{\mathbf{W}}\right)^{-1} \in \mathbf{L} .
$$

Moreover it holds that $q_{\mathbf{W}}^{-1} \cdot a \in \mathbf{W}$, and since

$$
q_{\mathbf{W}}^{-1} \cdot a \cdot a^{-1} \cdot q_{\mathbf{W}} \cdot q_{\mathbf{L}}^{-1} \cdot q_{\mathbf{W}}^{-1} \cdot a=q^{-1} \cdot a,
$$

the equation (4) holds and then (5) is a consequence of (4) and (3).

For the notion of intrinsically Lipschitz function we refer the reader to [9]. We explicitly recall here, for the reader's benefit, only the notions of intrinsically linear and intrinsically differentiable functions. For these definitions, and the study of some properties related, we refer the reader to $[2,11,12,8,18,3]$. Eventually, in order to fix the notation, we recall the notion of Pansu differentiability, see also [17].

Definition 2.4. (Intrinsically linear function) The map $\ell: \mathbf{W} \rightarrow \mathbf{L}$ is said to be intrinsically linear if graph $(\ell)$ is a homogeneous subgroup of $\mathbf{G}$.

Definition 2.5. (Intrinsically differentiable function) Let $\phi: U \subseteq \mathbf{W} \rightarrow \mathbf{L}$ be a function with $U$ Borel in $\mathbf{W}$. Fix a density point ${ }^{1} a_{0} \in \mathcal{D}(U)$ of $U$, let $p_{0}:=$ $\phi\left(a_{0}\right)^{-1} \cdot a_{0}^{-1}$ and denote by $\phi_{p_{0}}: U_{p_{0}} \subseteq \mathbf{W} \rightarrow \mathbf{L}$ the shifted function introduced in Definition 2.3. We say that $\phi$ is intrinsically differentiable at $a_{0}$ if there is an intrinsically linear map $d^{\phi} \phi_{a_{0}}: \mathbf{W} \rightarrow \mathbf{L}$ such that

$$
\lim _{b \rightarrow e, b \in U_{p_{0}}} \frac{\left\|d^{\phi} \phi_{a_{0}}[b]^{-1} \cdot \phi_{p_{0}}(b)\right\|}{\|b\|}=0 .
$$

The function $d^{\phi} \phi_{a_{0}}$ is called the intrinsic differential of $\phi$ at $a_{0}$. Notice that one can take the limit in (7) because, if $a_{0} \in \mathcal{D}(U)$, then $e \in \mathcal{D}\left(U_{p_{0}}\right)$. This last claim comes from the invariant properties of Proposition 2.1.

Remark 2.2. (Intrinsic differentiability and tangent subgroups) Whenever the intrinsic differential introduced in Definition 2.5 exists, it is unique: see [8, Theorem 3.2.8]. Let us recall the notion of tangent subgroup to the graph of a function. If we fix $\phi: U \subseteq \mathbf{W} \rightarrow \mathbf{L}$, we say that a homogeneous subgroup $\mathbf{T}$ of $\mathbf{G}$ is a tangent subgroup to $\operatorname{graph}(\phi)$ at $a_{0} \cdot \phi\left(a_{0}\right)$ if the following facts hold:

(a) $\mathbf{T}$ is a complementary subgroup of $\mathbf{L}$;

\footnotetext{
${ }^{1}$ If $k:=\operatorname{dim}_{H}(\mathbf{W})$ is the Hausdorff dimension of $\mathbf{W}$, we say that $a_{0} \in U$ is a density point of $U$, and we write $a_{0} \in \mathcal{D}(U)$, if $\mathcal{H}^{k}\left\llcorner\mathbf{W}\left(U \cap B\left(a_{0}, r\right)\right) / \mathcal{H}^{k}\left\llcorner\mathbf{W}\left(B\left(a_{0}, r\right)\right) \rightarrow 1\right.\right.$ as $r$ goes to 0 .
} 
(b) The limit

$$
\lim _{\lambda \rightarrow \infty} \delta_{\lambda}\left(\left(a_{0} \cdot \phi\left(a_{0}\right)\right)^{-1} \cdot \operatorname{graph}(\phi)\right)=\mathbf{T},
$$

holds in the sense of Hausdorff convergence on compact subsets of $\mathbf{G}$.

We notice that what the authors prove in [8, Theorem 3.2.8] is the following: a function $\phi: U \subseteq \mathbf{W} \rightarrow \mathbf{L}$, with $U$ open, is intrinsically differentiable at $a_{0}$ if and only if $\operatorname{graph}(\phi)$ has a tangent subgroup $\mathbf{T}$ at $a_{0} \cdot \phi\left(a_{0}\right)$ and moreover $\mathbf{T}=\operatorname{graph}\left(d^{\phi} \phi_{a_{0}}\right)$.

Definition 2.6. (Pansu differentiability) Let $\mathbf{W}$ and $\mathbf{G}$ be two arbitrary graded groups endowed with two homogeneous left-invariant distances $d_{\mathbf{W}}$ and $d_{\mathbf{G}}$, respectively. Given $f: U \subseteq \mathbf{W} \rightarrow \mathbf{G}$ with $U$ Borel and a density point $a_{0} \in \mathcal{D}(U)$, we say that $f$ is Pansu differentiable at $a_{0}$ if there exists a homogeneous homomorphism $d f_{a_{0}}: \mathbf{W} \rightarrow \mathbf{G}$, that we call Pansu differential at $a_{0}$, such that

$$
\lim _{a \rightarrow a_{0} a \in U} \frac{d_{\mathbf{G}}\left(f\left(a_{0}\right)^{-1} \cdot f(a), d f_{a_{0}}\left[a_{0}^{-1} \cdot a\right]\right)}{d_{\mathbf{W}}\left(a, a_{0}\right)}=0 .
$$

\section{Proof of the theorem}

In what follows we prove that, in case $\mathbf{L}$ is normal, the intrinsic differentiability of a function $\phi$ can be read as the Pansu differentiability of the graph map $\Phi$. For the forthcoming lemma we also refer the reader to [8, second item of Corollary 3.1.4].

Lemma 3.1. Let $\mathbf{W}$ and $\mathbf{L}$ be two complementary subgroups of a Carnot group $\mathbf{G}$, with $\mathbf{L}$ normal. The map $\ell: \mathbf{W} \rightarrow \mathbf{L}$ is intrinsically linear if and only if the graph map of $\ell$, i.e., $L: \mathbf{W} \rightarrow \mathbf{G}$ defined as $L(w):=w \cdot \ell(w)$, is a homogeneous homomorphism.

The forthcoming proposition is inspired by [4, Proposition 3.25(i)]. We give a detailed proof in our context, since the last part of the argument, i.e., the part in which we invoke the forthcoming Lemma 3.3, is different with respect to the reference.

Proposition 3.2. Let $\mathbf{W}$ and $\mathbf{L}$ be two complementary subgroups of a Carnot group $\mathbf{G}$, with $\mathbf{L}$ normal. Let $\phi: U \subseteq \mathbf{W} \rightarrow \mathbf{L}$ be a function with $U$ Borel. Given a density point $a_{0} \in \mathcal{D}(U)$, we have that $\phi$ is intrinsically differentiable at $a_{0}$ if and only if the graph map $\Phi: U \subseteq \mathbf{W} \rightarrow \mathbf{G}$ is Pansu differentiable at $a_{0}$. Moreover if any of the previous two holds we have the following formula:

$$
d \Phi_{a_{0}}[w]=w \cdot d^{\phi} \phi_{a_{0}}[w], \quad \forall w \in \mathbf{W} .
$$

Proof. Let us first notice that $p_{0}:=\phi\left(a_{0}\right)^{-1} \cdot a_{0}^{-1}=a_{0}^{-1} \cdot a_{0} \cdot \phi\left(a_{0}\right)^{-1} \cdot a_{0}^{-1}$, and thus, from the fact that $\mathbf{L}$ is normal,

$$
\left(p_{0}\right)_{\mathbf{W}}=a_{0}^{-1}, \quad\left(p_{0}\right)_{\mathbf{L}}=a_{0} \cdot \phi\left(a_{0}\right)^{-1} \cdot a_{0}^{-1} .
$$

Let us assume that $\phi$ is intrinsically differentiable at $a_{0}$. We are going to prove that the graph map $\Phi$ is Pansu differentiable at $a_{0}$. From the intrinsic differentiability of $\phi$, we know that there exists the intrinsic differential $d^{\phi} \phi_{a_{0}}$ as in Definition 2.5, which is an intrinsically linear map by definition. We define its graph map

$$
d \Phi_{a_{0}}[w]:=w \cdot d^{\phi} \phi_{a_{0}}[w], \quad \forall w \in \mathbf{W} .
$$

From Lemma 3.1, it follows that the map $d \Phi_{a_{0}}$ is a homogeneous homomorphism. We show that it is the Pansu differential of the graph map $\Phi$. Indeed, let us take 
$w \in U$ and compute

$$
\begin{aligned}
& d \Phi_{a_{0}}\left[a_{0}^{-1} \cdot w\right]^{-1} \cdot \Phi\left(a_{0}\right)^{-1} \cdot \Phi(w) \\
& =d^{\phi} \phi_{a_{0}}\left[a_{0}^{-1} \cdot w\right]^{-1} \cdot w^{-1} \cdot a_{0} \cdot \phi\left(a_{0}\right)^{-1} \cdot a_{0}^{-1} \cdot w \cdot \phi(w) \\
& =d^{\phi} \phi_{a_{0}}\left[a_{0}^{-1} \cdot w\right]^{-1} \cdot \phi_{p_{0}}\left(a_{0}^{-1} \cdot w\right),
\end{aligned}
$$

where in the first equality we used the definition (9) and in the second one we used (8) and the explicit expression in the first equality of (5). Notice that, from the second equality of (5) and the first equality of (8), we get that $U_{p_{0}}=a_{0}^{-1} \cdot U$. Thus (10), jointly with the intrinsic differentiability of $\phi$, see (7), tells us that

$$
\lim _{w \rightarrow a_{0}, w \in U} \frac{\left\|d \Phi_{a_{0}}\left[a_{0}^{-1} \cdot w\right]^{-1} \cdot \Phi\left(a_{0}\right)^{-1} \cdot \Phi(w)\right\|}{\left\|a_{0}^{-1} \cdot w\right\|}=0,
$$

that is the Pansu differentiability of $\Phi$ at $a_{0}$ with differential $d \Phi_{a_{0}}$.

Vice versa, let us assume that the graph map $\Phi$ is Pansu differentiable at $a_{0}$. Thus there exists a homogeneous homomorphism $d \Phi_{a_{0}}: \mathbf{W} \rightarrow \mathbf{G}$ such that

$$
\lim _{w \rightarrow a_{0}, w \in U} \frac{\left\|d \Phi_{a_{0}}\left[a_{0}^{-1} \cdot w\right]^{-1} \cdot \Phi\left(a_{0}\right)^{-1} \cdot \Phi(w)\right\|}{\left\|a_{0}^{-1} \cdot w\right\|}=0 .
$$

By using the fact that $\mathbf{L}$ is normal, and by simple computations, we get that

$$
\pi_{\mathbf{W}}\left(d \Phi_{a_{0}}\left[a_{0}^{-1} \cdot w\right]^{-1} \cdot \Phi\left(a_{0}\right)^{-1} \cdot \Phi(w)\right)=\left(\left(d \Phi_{a_{0}}\left[a_{0}^{-1} \cdot w\right]\right)_{\mathbf{W}}\right)^{-1} \cdot a_{0}^{-1} \cdot w, \quad \forall w \in U .
$$

Now notice that since there exists a geometric constant $C>0$ such that $\left\|g_{\mathbf{W}}\right\| \leq$ $C\|g\|$ for every $g \in \mathbf{G}$ (see [9, Proposition 2.12]), from the previous equality and (11) we deduce

$$
\lim _{w \rightarrow a_{0}, w \in U} \frac{\left\|\left(\left(d \Phi_{a_{0}}\left[a_{0}^{-1} \cdot w\right]\right) \mathbf{w}\right)^{-1} \cdot a_{0}^{-1} \cdot w\right\|}{\left\|a_{0}^{-1} \cdot w\right\|}=0 .
$$

Since $d \Phi_{a_{0}}$ is a homogeneous homomorphism, the limit in (12) allows us to use the forthcoming Lemma 3.3 with $U_{e}:=a_{0}^{-1} \cdot U$. This leads to the equality $\pi_{\mathbf{W}} \circ\left(d \Phi_{a_{0}}\right)=$ $(\text { id })_{\mid \mathbf{w}}$.

Thus, by using the splitting, for every $w \in \mathbf{W}$ it holds $d \Phi_{a_{0}}[w]=: w \cdot d^{\phi} \phi_{a_{0}}[w]$ for some map $d^{\phi} \phi_{a_{0}}: \mathbf{W} \rightarrow \mathbf{L}$. Now we are in a position to apply Lemma 3.1 to deduce that $d^{\phi} \phi_{a_{0}}$ is intrinsically linear, because its graph is a homogeneous homomorphism being a Pansu differential. Now the intrinsic differentiability of $\phi$ at $a_{0}$, with intrinsic differential $d^{\phi} \phi_{a_{0}}$, follows by joining the computations in (10) with (11).

Lemma 3.3. Let $\mathbf{W}$ and $\mathbf{L}$ be two complementary subgroups of a Carnot group $\mathbf{G}$ and let $U_{e} \subseteq \mathbf{W}$ be a Borel set containing the identity $e$ and for which $e$ is a density point. Let us assume $F: \mathbf{W} \rightarrow \mathbf{G}$ is a continuous homogeneous map, i.e., it commutes with $\delta_{\lambda}$ for every $\lambda>0$. Let us further assume that

$$
\lim _{w \rightarrow e, w \in U_{e}} \frac{\left\|(F(w) \mathbf{W})^{-1} \cdot w\right\|}{\|w\|}=0
$$

Then $F(w)_{\mathbf{W}}=w$ for every $w \in \mathbf{W}$.

Proof. Notice first that the map $w \rightarrow\left(F(w)_{\mathbf{W}}\right)^{-1}$ is a homogeneous map. Indeed, from the homogeneity of $F$, the homogeneity of the projection onto $\mathbf{W}$ and the 
homogeneity of the inverse, respectively, we get

$$
\begin{aligned}
\left(\left(F\left(\delta_{\lambda} w\right)\right)_{\mathbf{W}}\right)^{-1} & =\left(\left(\delta_{\lambda} F(w)\right)_{\mathbf{W}}\right)^{-1}=\left(\delta_{\lambda}\left(F(w)_{\mathbf{W}}\right)\right)^{-1} \\
& =\delta_{\lambda}\left(\left(F(w)_{\mathbf{W}}\right)\right)^{-1}, \quad \forall \lambda>0 .
\end{aligned}
$$

Set $S^{\mathbf{W}}:=\{w \in \mathbf{W}:\|w\|=1\}$. Since $e$ is a density point of $U_{e} \subseteq \mathbf{W}$, the following holds: there exists $D \subseteq S^{\mathbf{W}}$, dense in $S^{\mathbf{W}}$, such that for every $w \in D$ there exists an infinitesimal sequence $\left\{t_{j}\right\}_{j \in \mathbf{N}}$ such that $\delta_{t_{j}} w \in U_{e}$ for all $j \in \mathbf{N} .^{2}$

Fix $w \in D$. We claim that $F(w)_{\mathbf{W}}=w$ so that by density of $D$ and by the continuity and the homogeneity of $F$ the thesis follows. Indeed, let us fix $\epsilon>0$. By hypothesis, and since there exists an infinitesimal sequence $\left\{t_{j}\right\}_{j \in \mathbf{N}}$ such that $\delta_{t_{j}} w \in U_{e}$, we get that there is $t_{j_{0}}>0$ such that

$$
\left\|\left(F\left(\delta_{t_{j_{0}}} w\right)_{\mathbf{W}}\right)^{-1} \cdot \delta_{t_{j_{0}}} w\right\| \leq \epsilon\left\|\delta_{t_{j_{0}}} w\right\| .
$$

By the homogeneity in (13), the homogeneity of the norm and the fact that $\|w\|=1$, since $w \in D \subseteq S^{\mathbf{W}}$, we get

$$
\left\|\left(F(w)_{\mathbf{W}}\right)^{-1} \cdot w\right\| \leq \epsilon .
$$

Thus from the fact that $\epsilon>0$ is arbitrary we get the sought conclusion.

We are now in a position to prove our Theorem 1.1. Let us recall the definition of the Jacobian of a homogeneous map. Take a homogeneous map $F: \mathbf{W} \rightarrow \mathbf{G}$ between graded groups $\mathbf{W}$ and $\mathbf{G}$, equipped with homogeneous left-invariant distances $d_{\mathbf{W}}$ and $d_{\mathbf{G}}$, respectively. Denote by $k:=\operatorname{dim}_{H}(\mathbf{W})$ the Hausdorff dimension of $\mathbf{W}$. The Jacobian of $F$ is

$$
J(F):=\frac{\mathcal{H}^{k}(F(B(e, 1)))}{\mathcal{H}^{k}(B(e, 1))}
$$

where $B(e, 1)$ is the ball centered at the identity $e$ of $\mathbf{W}$, and of radius 1 .

Proof of Theorem 1.1. Since $\mathbf{L}$ is normal, we can use [9, Proposition 3.7] and thus, from the fact that $\phi$ is intrinsically Lipschitz, we deduce that the graph map $\Phi: U \subseteq \mathbf{W} \rightarrow \mathbf{G}$ is Lipschitz. Since $\mathbf{W}$ is a Carnot subgroup, due to Remark 2.1, we are in a position to apply Rademacher theorem (see [17] and [15, Theorem 3.9]) to the graph map $\Phi: U \subseteq \mathbf{W} \rightarrow \mathbf{G}$, in order to conclude that it is $\mathcal{H}^{k}\llcorner\mathbf{W}$-almost everywhere Pansu differentiable on $U$. Eventually, we apply Proposition 3.2 to conclude that every point of Pansu differentiability of $\Phi$ is a point of intrinsic differentiability of $\phi$. Finally, the area formula (1) is a direct consequence of the area formula in [16, Theorem 4.3.4] applied to the graph map $\Phi$, after having noticed that $\Phi: U \subseteq \mathbf{W} \rightarrow$ $\mathbf{G}$ is injective.

Remark 3.1. By joining the result of Theorem 1.1 and Remark 2.2 we conclude that in the hypotheses of Theorem 1.1 the intrinsically Lipschitz property guarantees the existence, at $(\Phi)_{*}\left(\mathcal{H}^{k}\llcorner U)\right.$-almost every point on the graph of $\phi$, of a tangent subgroup. Moreover, from Proposition 3.2 we get that, whenever the Pansu differential $d \Phi_{x}$ exists, then $\phi$ is intrinsically differentiable at $x$ and $d \Phi_{x}[w]=w \cdot d^{\phi} \phi_{x}[w]$ for all $w \in \mathbf{W}$. Taking into account this equality, the definition of the Jacobian (14) and Remark 2.2 we stress that the area element $J\left(d \Phi_{x}\right)$ in the area formula (1)

\footnotetext{
${ }^{2}$ Indeed if $U \subseteq \mathbf{W}$ is Borel and for $\mathcal{H}^{k}\llcorner\mathbf{W}$-almost every point $x$ in $U$ there exist a sequence $h_{j}(x)$ converging to $e$ and $0<\lambda(x)<1$ with $B\left(x \cdot h_{j}(x), \lambda(x)\left\|h_{j}(x)\right\|\right) \cap E=\emptyset$ for all $j \in \mathbf{N}$, then $\mathcal{H}^{k}\llcorner\mathbf{W}(E)=0$.
} 
only depends on the geometry of the tangent subgroup of graph $(\phi)$ at $\Phi(x)$, which is $\operatorname{graph}\left(d^{\phi} \phi_{x}\right)$.

Remark 3.2. The hypotheses of Theorem 1.1 are satisfied whenever we take an intrinsically Lipschitz function $\phi: U \subseteq \mathbf{W} \rightarrow \mathbf{L}$, with $\mathbf{W}$ horizontal. Thus our result applies in particular to intrinsically Lipschitz horizontal surfaces in arbitrary Carnot groups.

Remark 3.3. If we do not assume $\mathbf{L}$ to be normal in the hypotheses of Theorem 1.1, but still we assume that $\mathbf{W}$ is a Carnot subgroup, the graph map $\Phi: U \subseteq$ $\mathbf{W} \rightarrow \mathbf{L}$ may not be Lipschitz when $\phi$ is intrinsically Lipschitz. The forthcoming example is also found in [9].

Indeed, let us take the second Heisenberg group $\mathbf{H}^{2}$, with a basis of the Lie algebra given by $\left(X_{1}, X_{2}, X_{3}, X_{4}, X_{5}\right)$, where the only nontrivial relations are $\left[X_{1}, X_{3}\right]=$ $\left[X_{2}, X_{4}\right]=X_{5}$. Let us identify $\mathbf{H}^{2}$ with $\mathbf{R}^{5}$ by means of exponential coordinates of the first kind. Set, in those exponential coordinates, $\mathbf{W}:=\left\{\left(0, x_{2}, x_{3}, x_{4}, x_{5}\right): x_{2}, x_{3}, x_{4}\right.$, $\left.x_{5} \in \mathbf{R}\right\}$ and $\mathbf{L}:=\left\{\left(x_{1}, 0,0,0,0\right): x_{1} \in \mathbf{R}\right\}$. We notice that $\mathbf{W}$ is a Carnot subgroup and $\mathbf{L}$ is not normal.

It is easily verified that the map $\phi: \mathbf{W} \rightarrow \mathbf{L}$ defined as $\phi\left(0, x_{2}, x_{3}, x_{4}, x_{5}\right):=$ $(1,0,0,0,0)$ for every $\left(x_{2}, x_{3}, x_{4}, x_{5}\right) \in \mathbf{R}^{4}$ is intrinsically Lipschitz. Moreover, if we fix $\epsilon>0$ we have that $\Phi(0,0, \epsilon, 0,0)=(1,0, \epsilon, 0,-\epsilon / 2)$ and $\Phi(0,0,0,0,0)=(1,0,0,0,0)$. Thus

$$
\left\|\Phi(0,0,0,0,0)^{-1} \cdot \Phi(0,0, \epsilon, 0,0)\right\|=\|(0,0, \epsilon, 0,-\epsilon)\| \sim_{\epsilon \rightarrow 0} \epsilon^{1 / 2},
$$

and then $\Phi$ cannot be Lipschitz, since $\|(0,0, \epsilon, 0,0)\| \sim_{\epsilon \rightarrow 0} \epsilon$.

\section{References}

[1] Ambrosio, L., and B. Kirchheim: Rectifiable sets in metric and Banach spaces. - Math. Ann. 318:3, 2000, 527-555.

[2] Ambrosio, L., F. Serra Cassano, and D. Vittone: Intrinsic regular hypersurfaces in Heisenberg groups. - J. Geom. Anal. 16:2, 2006, 187-232.

[3] Antonelli, G., D. Di Donato, S. Don, and E. Le Donne: Characterizations of uniformly differentiable co-horizontal intrinsic graphs in Carnot groups. - Preprint, arXiv:2005.11390.

[4] Arena, G., and R. Serapioni: Intrinsic regular submanifolds in Heisenberg groups are differentiable graphs. - Calc. Var. Partial Differential Equations 35:4, 2009, 517-536.

[5] Chousionis, V., K. Fässler, and T. Orponen: Intrinsic Lipschitz graphs and vertical $\beta$ numbers in the Heisenberg group. - Amer. J. Math. 141:4, 2019, 1087-1147.

[6] Corwin, L. J., and F.P. Greenleaf: Representations of nilpotent Lie groups and their applications. Part I. Basic theory and examples. - Cambridge Stud. Adv. Math. 18, Cambridge Univ. Press, Cambridge, 1990.

[7] Federer, H.: Geometric measure theory. - Grundlehren Math. Wiss. 153, Springer-Verlag New York Inc., New York, 1969.

[8] Franchi, B., M. Marchi, and R. P. Serapioni: Differentiability and approximate differentiability for intrinsic Lipschitz functions in Carnot groups and a Rademacher theorem. - Anal. Geom. Metr. Spaces 2:1, 2014, 258-281.

[9] Franchi, B., and R. P. Serapioni: Intrinsic Lipschitz graphs within Carnot groups. - J. Geom. Anal. 26:3, 2016, 1946-1994.

[10] Franchi, B., R. Serapioni, and F. Serra Cassano: On the structure of finite perimeter sets in step 2 Carnot groups. - J. Geom. Anal. 13:3, 2003, 421-466.

[11] Franchi, B., R. Serapioni, and F. Serra Cassano: Intrinsic Lipschitz graphs in Heisenberg groups. - J. Nonlinear Convex Anal. 7:3, 2006, 423-441. 
[12] Franchi, B., R. Serapioni, and F. Serra Cassano: Differentiability of intrinsic Lipschitz functions within Heisenberg groups. - J. Geom. Anal. 21:4, 2011, 1044-1084.

[13] Julia, A., S. Nicolussi Golo, and D. VitTone: Area of intrinsic graphs and coarea formula in Carnot groups. - Preprint, arXiv:2004.02520.

[14] Le Donne, E.: A primer on Carnot groups: homogenous groups, Carnot-Carathéodory spaces, and regularity of their isometries. - Anal. Geom. Metr. Spaces 5:1, 2017, 116-137.

[15] Magnani, V.: Differentiability and area formula on stratified Lie groups. - Houston J. Math. 27:2, 2001, 297-323.

[16] Magnani, V.: Elements of geometric measure theory on sub-Riemannian groups. - Scuola Normale Superiore, Pisa, 2002.

[17] Pansu, P.: Métriques de Carnot-Carathéodory et quasiisométries des espaces symétriques de rang un. - Ann. of Math. (2) 129:1, 1989, 1-60.

[18] Serra Cassano, F.: Some topics of geometric measure theory in Carnot groups. - In: Geometry, analysis and dynamics on sub-Riemannian manifolds. Volume 1. - EMS Ser. Lect. Math., Eur. Math. Soc., Zürich, 2016, 1-121.

[19] Vittone, D.: Lipschitz graphs and currents in Heisenberg groups. - Preprint, arXiv:2007.14286.

Received 13 June $2020 \bullet$ Accepted 21 August 2020 